\title{
Galactorrhea Distressing Side Effects of Amisulpride
}

\author{
Jisha Myalil Lucca \\ Assistant Professor, College of Clinical Pharmacy Dpt Pharmaceutical Chemistry. Saudi Arabia \\ *Corresponding Author: Jisha Myalil Lucca, Assistant Professor, College of Clinical Pharmacy Dpt \\ Pharmaceutical Chemistry. Saudi Arabia
}

\begin{abstract}
Galactorrhea a distressing side-effect of conventional and second-generation antipsychotic drugs. 1 Hyperprolactinemia common in patients receiving amisulpride at high dose. Here we are presenting a patient with galactorrhea at a dose $25 \mathrm{mg}$ amisulpride that resolved after stopping the drug. Also patients psychiatric conditions are managed with aripiprazole
\end{abstract}

Keywords: Amisulpride, aripiprazole, Galactorrhea, side effects

\section{INTRODUCTION}

Hyperprolactinemia is a common and distressing side-effect of conventional and second-generation antipsychotic drugs. ${ }^{1}$ A prevalence of $65.6 \%$ has been reported in premenopausal women treated with different antipsychotics, among which risperidone had a greater likelihood of inducing hyperprolactinemia than the conventional antipsychotics. ${ }^{1}$ Of all the SGA risperidone and amisulpride have the highest propensity to elevate prolactin level. ${ }^{2}$ Elevated levels of prolactin in females cause menstrual disturbances and galactorrhea and have been linked with disturbed sexual function in terms of desire, erections, orgasm in females and may even cause hypogonadism. ${ }^{1,2}$ Ziprasidone seems to be less frequently associated with hyperprolactinemia, and aripiprazole may even lower prolactin level. ${ }^{3}$ This case report is being highlighted having hyperprolactinemia with a low dosage $(25 \mathrm{mg})$ of the novel antipsychotic drug Amisulpride.

\section{Case Study}

A 15 year old unmarried adolescent female with ICD-10 diagnosis of bipolar mood disorders and with a history of complex partial seizure was brought by her mother to the department of psychiatry. The illness had begun a year prior to this visit, when she started to ran away from home, wandering behaviors, decreased interaction and altered sleep. She was on treatment with risperidone $1 \mathrm{mg}$ one at night and sodium valproate $500 \mathrm{mg}$ BD. As there was no improvement in her psychopathology even after a month of treatment, risperidone was stopped and the patient was switched to amisulpride 25 $\mathrm{mg}$ one in the night. After 2 days of amisulpride treatment the patient complained of galactorrhea and breast tenderness. Patient denied sexual activity, trauma, or manipulation of her breasts. Thyroid function test and serum prolactin level could not be estimated as patient was not willing for the test. Gynecological opinion sort and was nothing significant.Amisulpride was stopped and the patient was switched to aripiprazole $10 \mathrm{mg} /$ day. Surprisingly, 3 days after the beginning of aripiprazole treatment, galactorrhea disappeared. On 6 months follow up the patient was still treated with aripiprazole $10 \mathrm{mg} /$ day without reoccurrence of galactorrhea and mental status remained stable and the patient was free from any adverse effects of medication.

\section{CASE Discussion}

Amisulpride, a substituted benzamide derivative, is a second-generation antipsychotic that preferentially binds to D2/D3 receptors in limbic rather than striatal structures. ${ }^{4}$ The effect on prolactin secretion is due to a blockade of pituitary action of the tuberoinfundibular dopaminergic system. Amisulpride has a potent prolactin-elevating effect, similar to conventional antipsychotic drugs. ${ }^{4}$

Aripiprazole is a potent partial agonist of the D2 receptors.It exhibits functional agonist properties under hypodopaminergic conditions but acts as functional antagonist at D2 receptors under 
hyperdopaminergic conditions. ${ }^{5}$ Aripiprazole may therefore inhibit prolactin secretion by blocking D2 receptors in the pituitary gland (where the dopamine neuronal activity is tonically controlled) and function as a dopaminergic agonist at low levels of dopaminergic neuronal activity. ${ }^{5,6}$ The normalization of prolactin concentration may be the result of discontinuing the prolactin-elevating antipsychotic agents. In contrast, aripiprazole did not negatively influence prolactin level in this highly susceptible female. ${ }^{6}$ The very rapid time course, however, of normalization ( 3 days), supports the hypothesis that aripiprazole has an independent effect on prolactin level. ${ }^{3}$ The present findings are consistent with reports indicating that aripiprazole reduces elevated prolactin level and resolves galactorrhea and amenorrhea. Controlled trial showed that adjunctive treatment with aripiprazole successfully alleviated hyperprolactinemia in schizophrenia patients maintained with haloperidol but there are also reports of aripiprazole-induced galactorrhea.

\section{CONClusion}

This report showed that switching to a prolactin-sparing antipsychotic is a more effective strategy for the resolution of amenorrhea, because of the risk that reducing the dosage of the antipsychotic either does not resolve the amenorrhea or results in the relapse of psychosis.

\section{REFERENCES}

[1] Haddad P, Wieck A. Antipsychotic-induced hyperprolactinaemia: mechanisms, clinical features and management.Drugs 2004; 64 (20):2291-2314

[2] Bun-Hee Lee, M.D., Yong-Ku Kim, M.D. Resolution of Amisulpride associated Amenorrhea by Switching to Aripiprazole Psychiatry Invest 2006; 3 (1):102-106

[3] Mixalis S, Georgios P, Konstantinos K, DimitriosKAripiprazole resolves amisulpride and ziprasidoneinduced Hyperprolactinemia Psychiatry and Clinical Neurosciences 2008; 62; 624

[4] Raj R, Sidhu BS. Hyperprolactinaemia with amisulpride. Indian J Psychiatry. 2008;50(1):54-6.

[5] Raveendranthan D, Rao NP, Rao MG, et al. Add-on Aripiprazole for Atypical Antipsychotic-induced, Clinically Significant Hyperprolactinemia. Indian J Psychol Med. 2018;40(1):38-40.

[6] Ranjbar F, Sadeghi-Bazargani H, Niari Khams P, Arfaie A, Salari A, Farahbakhsh M. Adjunctive treatment with aripiprazole for risperidone-induced hyperprolactinemia. Neuropsychiatr Dis Treat. 2015;11:549-55.

Citation: Myalil Lucca j, (2019). Galactorrhea Distressing Side Effects of Amisulpride. ARC Journal of Pharmaceutical Sciences (AJPS), 5(1), pp.5-6. http://dx.doi.org/10.20431/2455-1538.0501002

Copyright: () 2019 Authors. This is an open-access article distributed under the terms of the Creative Commons Attribution License, which permits unrestricted use, distribution, and reproduction in any medium, provided the original author and source are credited. 